\title{
Floral induction and monocarpic versus polycarpic life histories
}

\author{
Richard Amasino
}

Address: Department of Biocemistry, University of Wisconsin, Babcock Drive, Madison, WI 53706-1544, USA. Email: amasino@biochem.wisc.edu.

\begin{abstract}
Asbtract
Recent work in Arabis alpina, a perennial relative of Arabidopsis, has uncovered subtle differences in control of a gene that represses flowering which contributes to the polycarpic habit.
\end{abstract}

There are two extremes of life-history strategies in plants and animals - semelparity and iteroparity [1]. Semelparity is sometimes referred to as the 'big-bang reproductive strategy' [2], as semelparous species devote most of their energy and resources to maximizing the number of offspring in a single cycle of reproduction, and die soon after reproducing. Semelparity may be advantageous when the prospects for long-term survival are low. Iteroparous species, in contrast, reproduce multiple times, a strategy that may be advantageous when prospects for long-term survival are good.

In the plant kingdom, there are extreme examples of both strategies. At one end of the iteroparous spectrum are redwood trees, which can live for several thousand years with several thousand cycles of reproduction. In contrast, the popular semelparous research model Arabidopsis thaliana can complete its life cycle in less than two months, and once Arabidopsis produces a certain number of offspring it rapidly senesces and dies, even under optimal growth conditions [3] (Figure 1).

Plants that live and reproduce for many years, such as redwoods, are often referred to as perennials. Plants such as Arabidopsis that typically survive only a single growing season are often referred to as annuals. However, the different life-history strategies of plants are better described by the terms monocarpic (semelparous; reproduces once and dies) and polycarpic (iteroparous; reproduces repeatedly), instead of annual and perennial, respectively. For example, perennial is hard to define, because there are plants that live for many years without flowering and then flower once and die. A striking example is the Haleakala silversword, Argyroxiphium sandwicense, which may live for more than 50 years before flowering and dying (Figure 1).

The molecular basis for the death of monocarpic plants like Arabidopsis after reproduction is not well understood.
Plants develop from regions of stem cells called meristems. The shoot apical meristem (SAM) produces cells that differentiate into stems, leaves and flowers. In many monocarpic plants, including Arabidopsis, all active SAMs convert to flower production (that is, become inflorescence meristems). In Arabidopsis, when a certain number of seeds have been produced the inflorescence meristems stop growing, although they do not undergo terminal differentiation, and the whole plant senesces as the seeds mature [3]. Perhaps inflorescence meristem arrest after reproduction and the subsequent death is a specific genetic program in Arabidopsis, or perhaps the plants simply do not have the energy to sustain further growth from these inflorescence meristems - the plants 'burn out' in the effort to produce as many offspring as possible [3].

Thus, a key feature of polycarpy is to maintain a supply of meristems that are capable of vegetative growth; that is, SAMs that can produce shoots with leaves to sustain growth of the plant in future growth cycles. In a recent paper in Nature by Wang et al. [4], the polycarpic habit was studied in a relative of Arabidopsis, Arabis alpina, another member of the family Brassicaceae. A. alpina requires exposure to cold in order to flower (a phenomenon known as vernalization) [5]. However, as expected for a polycarpic plant, vernalization does not result in the flowering of all $A$. alpina SAMs. Those shoots of $A$. alpina that do flower cease growth and senesce during seed maturation similarly to shoots of Arabidopsis, but $A$. alpina maintains a supply of vegetative SAMs for another round of growth.

\section{From polycarpy towards monocarpy}

Wang et al. [4] identified an A. alpina mutant, perpetual flowering 1 (pep1), that does not require vernalization for flowering. Furthermore, in non-vernalized pep1 mutants, a greater fraction of SAMs become inflorescence meristems than in vernalized wild-type plants. Therefore, PEP1 is required both to create a vernalization requirement and to ensure that a certain fraction of SAMs remain vegetative. Previous work in Arabidopsis has established that FLOWERING LOCUS C (FLC), a gene encoding a MADSdomain transcription factor, is a flowering repressor that prevents SAMs from flowering in the fall and creates a vernalization requirement [5]. Thus, Wang et al. [4] 


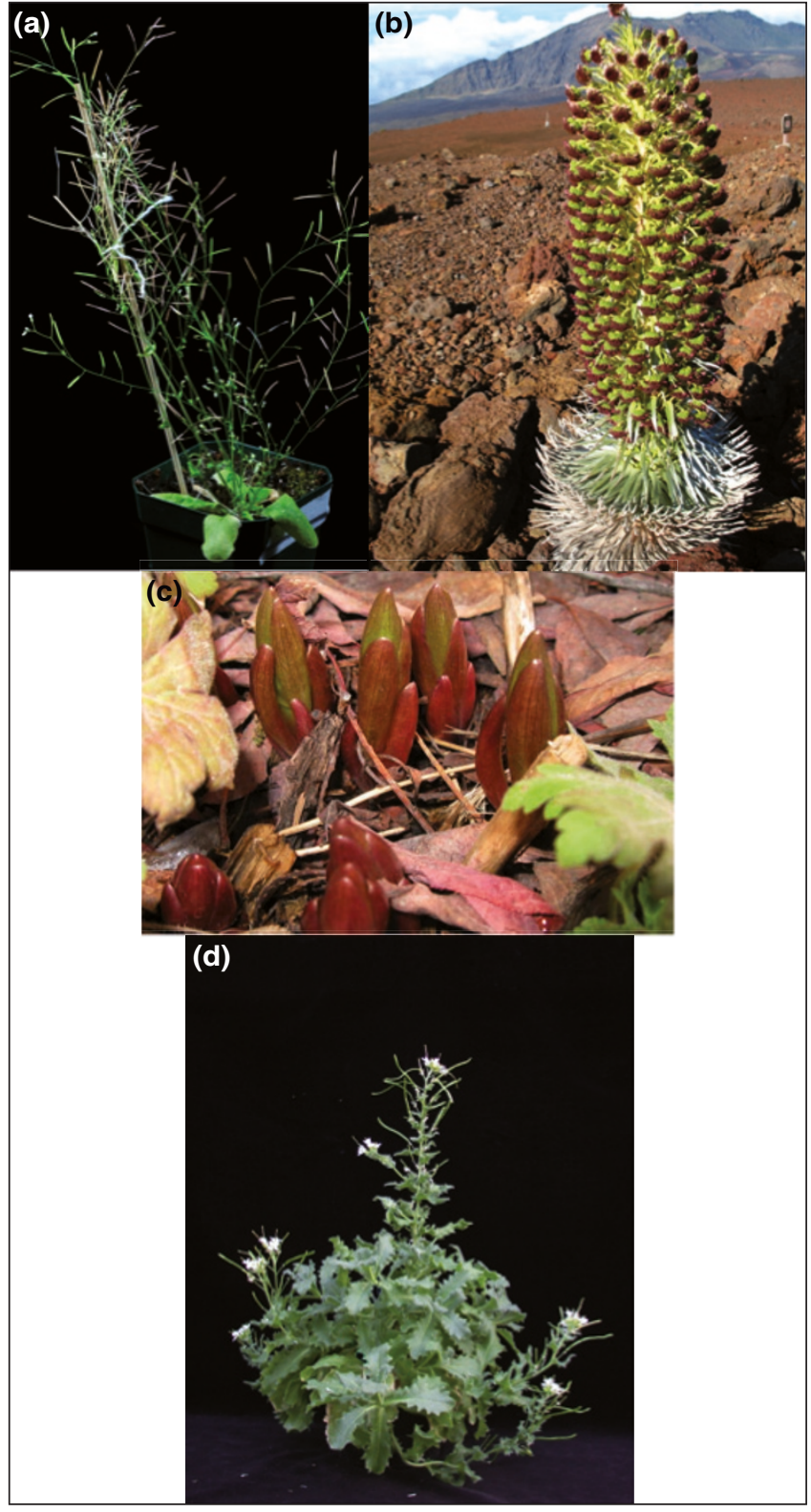

Figure 1

Examples of monocarpic and polycarpic plants. (a) A plant of Arabidopsis thaliana that has produced sufficient seed and is entering the phase of whole-plant senescence characteristic of many monocarpic plants. All of the shoots are floral, and this plant will soon die, despite being kept in optimal growth conditions. (b) Like $A$. thaliana, the monocarpic Haleakalā silversword dies after reproduction. But unlike $A$. thaliana, the silversword typically grows for several decades before flowering. (c) The above-ground parts of many polycarpic perennials that are adapted to temperate climates do senesce each year as winter approaches, and new growth emerges from below-ground parts of the plant in the following spring, as illustrated by this member of the lily family. (d) Arabis alpina is a polycarpic relative of $A$. thaliana. Whereas all shoots of $A$. thaliana undergo the floral transition, some $A$. alpina shoots remain vegetative to permit further growth and flowering in future years. A. alpina is a short-lived perennial that does not 'die back' in preparation for winter. Image of $A$. alpina courtesy of Maria Albani. hypothesized that PEP1 might be the A. alpina homolog of $F L C$, and demonstrated that this is indeed the case. What is interesting is that vernalization only transiently results in PEP1 repression in A. alpina; this is in contrast to the situation in Arabidopsis, in which vernalization can result in a stable repression of FLC [5]. Only those A. alpina SAMs that actually initiate flowers during cold exposure produce flowering shoots when warm temperatures return. Even quite long periods of cold exposure are not sufficient to convert all SAMs to flowering, and the resumption of FLC expression in the non-flowering SAMs in warm temperatures ensures that these SAMs remain vegetative and that A. alpina is polycarpic.

In Arabidopsis, the stability of $F L C$ repression is associated with repressive modifications to FLC chromatin, such as increased trimethylation of histone 3 at lysine 27 ( $\mathrm{H}_{3} \mathrm{~K} 27$ triMe) and lysine 9 ( $\mathrm{H}_{3} \mathrm{~K} 9$ triMe). These modifications are initiated during a vernalizing cold exposure, and the levels of these modifications increase after plants experience warm temperatures (see, for example, [6-10]). In A. alpina, $\mathrm{H}_{3} \mathrm{~K} 27$ triMe levels in PEP1 chromatin increase during cold, but then decrease when plants are returned to warm temperatures [4]. It will be interesting to explore the molecular basis of PEP1 expression and histone modification reversibility in A. alpina. For example, is reversibility inherent in the PEP1 locus (for example, might PEP1 lack certain cis-regulatory elements that are required for stable repression)? If this is the case, then PEP1 might exhibit a similar transient repression even when introduced into Arabidopsis. There are precedents for such 'cis effects'. Deletion of a region of the first intron of Arabidopsis $F L C$ known as the 'vernalization response element (VRE)' creates a 'PEP1-like' allele for which cold repression is not maintained [8], and vernalization-mediated repression of cabbage FLC may not be maintained when the gene is introduced into Arabidopsis [11]. Alternatively, PEP1 reversibility may be due to differences in the chromatinmodifying complexes in A. alpina compared with Arabidopsis; if this were the case, Arabidopsis FLC might be only transiently repressed in A. alpina. There are also precedents in Arabidopsis for this alternative. The reversible, cold-specific repression of PEP1 in A. alpina is similar to that observed for FLC in certain Arabidopsis mutants such as lhp1, vrn1 and vrn2 [6-8,12-14].

Regardless of the mechanism of PEP1 repression, it is clear that an important difference in the monocarpic versus polycarpic life histories of Arabidopsis versus A. alpina is, respectively, the permanent versus transient repression of FLC/PEP1 by vernalization. This is not the complete story, however. As Wang et al. [4] discuss, pep1 mutants do not phenocopy the monocarpic habit of Arabidopsis; some SAMs remain vegetative, and the pep1 mutant continues to grow indefinitely after flowering. This indicates that additional genes are responsible for the monocarpic habit. Perhaps a 
further round of mutagenesis in the pep1 mutant background might result in monocarpic lines, and thus reveal additional genes that are involved in life-history traits.

\section{From monocarpy towards polycarpy}

Looking at the question from another angle, Melzer et al. [15] reported in a paper in Nature Genetics last year that loss of two genes, SUPPRESSOR OF CONSTANS 1 (SOC1) and FRUITFULL (FUL), causes Arabidopsis to assume a polycarpic habit. As discussed earlier, the monocarpic habit in Arabidopsis is caused, at least in part, by conversion of all active SAMs into inflorescence meristems, which eventually stop growing (although they do not terminally differentiate, as implied in [15]). In wild-type Arabidopsis, once a SAM becomes floral it never reverts to vegetative growth because a positive feedback loop of floral promoters locks in the flowering state [16-18]. Melzer et al. [15] show that SOC1 and FUL are required for this lock-in. In soc1/ful double mutants, some inflorescence meristems revert to vegetative growth and other SAMs do not flower. The resulting double-mutant plants do not completely senesce after flowering because the vegetative SAMs keep growing.

Polycarpy requires not only the preservation of vegetative SAMs for future growth cycles, but the ability to produce new vascular tissue (secondary growth) to maintain the connection between shoots and the root system. In soc1/ful double mutants, there is enhanced secondary growth, and Melzer et al. suggest that 'loss of SOC1 and FUL function rather than the increased life span of the plants was responsible for the observed secondary growth' [15], but it is also possible that the enhanced secondary growth is an indirect effect of the presence of active vegetative SAMs in plants that are flowering. Vegetative SAMs on a flowering stem might, for example, alter phytohormone levels and fluxes such that secondary growth is favored.

Given that there are typically both monocarpic and polycarpic species within the same plant family, and that their relationships indicate that transitions between monocarpy and polycarpy are common, perhaps the genetic differences between monocarpic and polycarpic species in a particular family are not extensive. These recent studies are an exciting start towards understanding the genetic basis of the difference between monocarpic and polycarpic habits in the Brassicaceae.

\section{References}

1. Charlesworth B: Evolution in Age-Structured Populations. Cambridge, UK: Cambridge University Press; 1980.
2. Humphries S, Stevens DJ: Reproductive biology: Out with a bang. Nature 2001, 410:758-759.

3. Bleecker AB, Patterson SE: Last exit: Senescence, abscission, and meristem arrest in Arabidopsis. Plant Cell 1997, 9:1169-1179.

4. Wang R, Farrona S, Vincent C, Joecker A, Schoof H, Turck F, Alonso-Blanco C, Coupland G, Albani MC: PEP1 regulates perennial flowering in Arabis alpina. Nature 2009, 459:423427.

5. Amasino R: Vernalization, competence, and the epigenetic memory of winter. Plant Cell 2004, 16:2553-2559.

6. Bastow R: Vernalization requires epigenetic silencing of FLC by histone methylation. Nature 2004, 427:164-167.

7. Sung S, Amasino RM: Vernalization in Arabidopsis thaliana is mediated by the PHD finger protein VIN3. Nature 2004, 427:159-164.

8. Sung S, He Y, Eshoo TW, Tamada Y, Johnson L, Nakahigashi $\mathrm{K}$, Goto K, Jacobsen SE, Amasino R: Epigenetic maintenance of the vernalized state in Arabidopsis thaliana requires LIKE HETEROCHROMATIN PROTEIN 1. Nat Genet 2006, 38:706-710.

9. Finnegan EJ, Dennis ES: Vernalization-induced trimethylation of histone $\mathrm{H} 3$ lysine 27 at FLC is not maintained in mitotically quiescent cells. Curr Biol 2007, 17:1978-1983.

10. Sung SY, Schmitz RJ, Amasino RM: A PHD finger protein involved in both the vernalization and photoperiod pathways in Arabidopsis. Genes Dev 2006, 20:3244-3248.

11. Lin SI, Wang JG, Poon SY, Su Cl, Wang SS, Chiou TJ: Differential regulation of FLOWERING LOCUS C expression by vernalization in cabbage and Arabidopsis. Plant Physiol 2005, 137:1037-1048.

12. Mylne JS, Barrett L, Tessadori F, Mesnage S, Johnson L, Bernatavichute YV, Jacobsen SE, Fransz P, Dean C: LHP1, the Arabidopsis homologue of HETEROCHROMATIN PROTEIN1, is required for epigenetic silencing of FLC. Proc Natl Acad Sci USA 2006, 103:5012-5017.

13. Gendall AR, Levy YY, Wilson A, Dean C: The VERNALIZATION 2 gene mediates the epigenetic regulation of vernalization in Arabidopsis. Cell 2001, 107:525-535.

14. Levy YY, Mesnage S, Mylne JS, Gendall AR, Dean C: Multiple roles of Arabidopsis VRN1 in vernalization and flowering time control. Science 2002, 297:243-246.

15. Melzer S, Lens F, Gennen J, Vanneste S, Rohde A, Beeckman T: Flowering-time genes modulate meristem determinacy and growth form in Arabidopsis thaliana. Nat Genet 2008, 40:1489-1492.

16. Tooke F, Ordlidge M, Chiurugwi T, Battey N: Mechanisms and function of flower and inflorescence reversion. $J$ Exp Bot 2005, 56:2587-2599.

17. Hempel FD, Welch DR, Feldman LJ: Floral induction and determination: where is flowering controlled? Trends Plant Sci 2000, 5:17-21.

18. Ferrandiz C, Gu Q, Martienssen R, Yanofsky M: Redundant regulation of meristem identity and plant architecture by FRUITFULL, APETALA1 and CAULIFLOWER. Development 127:725-734.

Published: 2 July 2009

doi:10.1186/gb-2009-10-7-228

(C) 2009 BioMed Central Ltd 